Algebraic $8 \mathcal{G}$ Geometric $\mathcal{T}_{\text {opology }}$

Volume 4 (2004) 311-332

Published: 21 May 2004

ATG

\title{
Shadow world evaluation of the Yang-Mills measure
}

\author{
Charles Frohman \\ JOANNA KANIA-BARTOSZYNSKA
}

\begin{abstract}
A new state-sum formula for the evaluation of the Yang-Mills measure in the Kauffman bracket skein algebra of a closed surface is derived. The formula extends the Kauffman bracket to diagrams that lie in surfaces other than the plane. It also extends Turaev's shadow world invariant of links in a circle bundle over a surface away from roots of unity. The limiting behavior of the Yang-Mills measure when the complex parameter approaches -1 is studied. The formula is applied to compute integrals of simple closed curves over the character variety of the surface against Goldman's symplectic measure.
\end{abstract}

AMS Classification 57M27; 57R56, 81T13

Keywords Yang-Mills measure, shadows, links, skeins, $S U(2)$-characters of a surface

\section{Introduction}

The Kauffman bracket [8] is an unoriented framed version of the Jones polynomial of oriented links [7]. Kirilov and Reshetikhin gave a state sum formula for the Kauffman bracket [1] where the states are admissible colorings of the faces of the link diagram. This formalism was extended further to diagrams in surfaces other than the plane by Turaev, who then used it to give invariants of knots and links in circle bundles over a surface corresponding to roots of unity 15. This formula is known as shadow world evaluation.

Let $F$ be an oriented surface and let $t$ be a non-zero complex number. The Kauffman bracket skein $K_{t}(F)$ is an algebra derived from links in a cylinder over $F$. When $t=-1$ this algebra is isomorphic to the $S U(2)$-characters of the fundamental group of $F$ [1, [13. As $t$ moves away from -1 the deformation of the algebra 2] corresponds to the Poisson structure on the characters coming from Goldman's symplectic structure [6].

In 3] we constructed a diffeomorphism invariant trace $\mathcal{Y} \mathcal{M}: K_{t}(F) \rightarrow \mathbb{C}$, called the Yang-Mills measure, that is defined for all $t$ with $|t| \neq 1$ and when $t$ is a 
root of unity. When $t=-1$ this trace coincides with integration against the symplectic measure on the variety underlying the $S U(2)$-characters of $\pi_{1}(F)$. The existence of the trace was established by a study of its behavior on admissibly colored travalent spines of the surface. In this paper we recognize that the Yang-Mills measure can be computed using a state-sum formula which corresponds exactly to the formulas used by Kirilov and Reshetikhin and later by Turaev.

The power of the shadow world formula is that it places the computation of the Jones polynomial and integration on the $S U(2)$-character variety in the same framework. Using the combinatorial data from a link diagram in the surface, we can quickly perform computations that were before quite difficult. For instance, we prove that integrating a simple non-separating curve over the character variety of a surface always yields zero. We also obtain values of the integrals along simple separating curves on a closed surface of genus bigger than one.

After establishing the shadow world formula for the Yang-Mills measure, we use it together with recoupling theory to compute the limit of the trace at roots of unity as the order of the root tends towards infinity. When $F$ is a closed surface of genus bigger than 1 it converges to twice Goldman's symplectic measure, i.e., to twice the classical Yang-Mills measure.

When the complex parameter $t$ is a root of unity, this formula coincides with Turaev's shadow world evaluation for links in a circle bundle over $F$ with Euler number zero [15]. We investigate the extent to which Turaev's shadow world evaluation of links in nontrivial circle bundles can be extended away from roots of unity. It turns out that the convergence depends on the Euler class of the bundle.

The shadow world evaluation also resembles the formula for the Turaev-Viro invariants. The estimates in this paper led us to initiate the study of the Turaev-Viro invariants of three-manifolds away from roots of unity [5]. This formula can also be used to investigate the relationship between the TuraevViro invariant and the Reidemeister torsion of the $S U(2)$-representations of the fundamental group of the 3-manifold [4.

This paper is organized as follows. Section 2 contains definitions and recalls from [3] the construction of the Yang-Mills measure away from roots of unity. Section 3 derives the state sum formula for the Yang-Mills measure. In section 4 we investigate the extension of Turaev's shadow invariant of links in circle bundles over a surface. In section 5 we analyze the limit of the Yang-Mills 
measure as the order of roots of unity goes to infinity. Finally in the last section some sample integrals on the character variety are evaluated.

This research was partially supported by NSF grants DMS-0207030 and DMS0204627.

\section{Kauffman bracket skein algebra of a surface}

\subsection{Preliminaries}

Let $F$ be a compact orientable surface (with or without boundary). The cylinder over $F$ is the three-manifold $F \times[0,1]$. A framed link in the cylinder over $F$ is an embedding of a disjoint union of annuli into $F \times[0,1]$. Two framed links are equivalent if there is an isotopy of $F \times[0,1]$ taking one to the other. Framed links in $F \times[0,1]$ can be visualized as diagrams on the surface $F$. A diagram is an isotopy class of four-valent graphs in $F$ with a marking at each vertex to indicate which strand goes over and which strand goes under. The embedded annuli corresponding to the diagram come from gluing together strips that run parallel to the surface with the edges of the graph as their core, glued together in $F \times[0,1]$, according to the over and under-crossing data. The isotopy class of any framed link can be represented this way, and two diagrams represent the same framed link if and only if they differ by the second and third Reidemeister moves along with the move

$$
\rho=\alpha
$$

Let $\mathcal{L}$ denote the set of isotopy classes of framed links in $F \times[0,1]$, including the empty link. Fix a complex number $t \neq 0$. Consider the vector space $\mathbb{C} \mathcal{L}$ with basis $\mathcal{L}$. Let $S(F)$ be the smallest subspace of $\mathbb{C} \mathcal{L}$ containing all expressions of the form $\backslash-t \smile-t^{-1}$ ) ( and $\bigcirc \cup L+\left(t^{2}+t^{-2}\right) L$. The first relation means that there are three framed links and a ball inside $F \times[0,1]$. The three links coincide outside the ball, however, the links intersect the ball as blackboard framed arcs as shown in the relation. The second relation means that there are two framed links and a ball in the manifold, so that outside the ball the two framed links coincide, however one link misses the ball and the other intersects it in a 0 -framed unknot. The Kauffman bracket skein $K_{t}(F)$ is the quotient

$$
\mathbb{C} \mathcal{L} / S(F) .
$$

The algebra structure on $K_{t}(F)$ comes from laying one link over the other. Suppose that $\alpha, \beta \in K_{t}(F)$ are skeins represented by links $L_{\alpha}$ and $L_{\beta}$. After 
isotopic deformations, to "lower" the first link and "raise" the second, $L_{\alpha} \subset$ $F \times\left[0, \frac{1}{2}\right)$, and $L_{\beta} \subset F \times\left(\frac{1}{2}, 1\right]$. The skein $\alpha * \beta$ is represented by $L_{\alpha} \cup L_{\beta}$. This can be extended using the distributive law to a product on $K_{t}(F)$.

The notation and the formulas in this paper are taken from [9, ch. 9]. However, the variable $t$ replaces $A$, and we use quantum integers

$$
[n]=\frac{t^{2 n}-t^{-2 n}}{t^{2}-t^{-2}}
$$

When $t= \pm 1,[n]=n$.

There is a standard convention, 9, ch. 4], for modeling a skein in $K_{t}(F)$ on a framed trivalent graph $\Gamma \subset F$. An admissible coloring of $\Gamma$ is an assignment of a nonnegative integer to each edge of $\Gamma$ so that the colors at trivalent vertices form admissible triples. A triple $(a, b, c)$ is admissible if $a+b+c$ is even, $a \leq b+c, b \leq a+c$, and $c \leq a+b$. Viewing $F$ as $F \times\left\{\frac{1}{2}\right\}$, the skein in $K_{t}(F)$ corresponding to an admissibly colored graph in $F$ is obtained by replacing each edge labeled with the letter $m$ by the $m$-th Jones-Wenzl idempotent (see [16, 9, ch. 3] or [12, p.136]), and replacing trivalent vertices with Kauffman triads (see [9, ch. 4], 12, Fig. 14.7]). If $F$ has nonempty boundary and $\gamma$ is a trivalent spine of $F$, then the collection of skeins modeled on all admissible colorings of $\gamma$ forms a basis for $K_{t}(F)$.
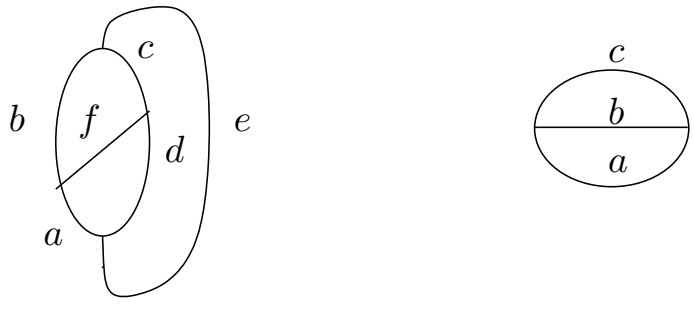

Figure 1: Tet and theta

We often use values of the Kauffman bracket of the following three skeins in a disk. The zero framed unknot colored with the $n$-th Jones-Wenzl idempotent, denoted by $\Delta_{n}$, is equal to $(-1)^{n}[n+1]$. The symbol Tet $\left(\begin{array}{lll}a & b & e \\ c & d & f\end{array}\right)$ stands for the bracket of the skein modeled by the colored graph pictured in Figure 1 on the left. Finally, the quantity $\theta(a, b, c)$ is the bracket of the skein pictured on the right in Figure 11 The explicit formulas are given in [9, pp.97-8]. 
Recall the fusion identity:

$$
\left.\right|^{a}=\sum_{c} \frac{\Delta_{c}}{\theta(a, b, c)} \sum_{c}^{a}{ }_{b}^{b}
$$

where the sum is over all $c$ so that the triples $(a, b, c)$ are admissible. The fusion relation is satisfied in $K_{t}(F)$ unless $t$ is a root of unity other than \pm 1 .

When $t=e^{\frac{\pi i}{2 r}}$ for odd $r>1$ we work in the quotient of $K_{t}(F)$ where the appropriate form of the fusion identity has been enforced. In this case triples are $r$-admissible, that is $a+b+c \leq 2 r-4$, in addition to the previous conditions. Dividing by the fusion relation is equivalent to taking a quotient of $K_{t}(F)$ by the submodule spanned by skeins corresponding to trivalent graphs where some edge carries the label $r-1$ [14]. We denote the quotient algebra $K_{r}(F)$. Just as admissibly colored spines of $F$ form a basis for $K_{t}(F), r$-admissibly colored spines are a basis for $K_{r}(F)$. If $F$ is closed, $K_{r}(F)$ is isomorphic to $M_{n}(\mathbb{C})$ for some $n$ that depends on $r$ and on the topological type of the surface [14].

The $6 j$-symbol is defined by

$$
\left\{\begin{array}{lll}
a & b & e \\
c & d & f
\end{array}\right\}=\frac{\operatorname{Tet}\left(\begin{array}{lll}
a & b & e \\
c & d & f
\end{array}\right) \Delta_{e}}{\theta(a, d, e) \theta(b, c, e)} .
$$

The recoupling formula says that:

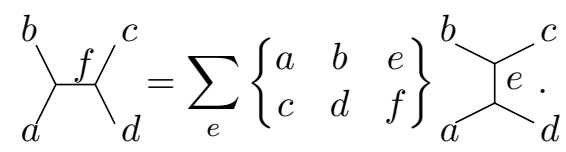

All of the quantities $\Delta_{a}, \theta(a, b, c)$,

$$
\operatorname{Tet}\left(\begin{array}{lll}
a & b & e \\
c & d & f
\end{array}\right) \text {, and }\left\{\begin{array}{lll}
a & b & e \\
c & d & f
\end{array}\right\}
$$

may fail to be defined at $4 r$ th roots of unity when one of the arguments is bigger than $r-2$. However, fixing the arguments, and viewing them as functions of $t$, they are all continuous in neighborhoods of $t=-1$.

Given a link $L \subset F \times I$ and a spine $\gamma$ of $F$, for each $t$ (and for each $r$ ) $L$ can be written as a linear combination of skeins corresponding to admissible (and respectively $r$-admissible) colorings of the spine. These coefficient are not necessarily continuous as $t$ approaches $e^{\frac{\pi i}{2 r}}$. However, they are continuous at $t=-1$. 


\section{$2.2 \quad$ Yang-Mills measure}

Suppose that $F$ is a closed surface of genus $g$. Since the quotient of $M_{n}(\mathbb{C})$ by the subvector space of commutants is one dimensional there is a unique trace $\mathcal{Y} \mathcal{M}: K_{r}(F) \rightarrow \mathbb{C}$ so that $\mathcal{Y} \mathcal{M}(\emptyset)=\sum_{i=0}^{r-2} \frac{1}{[i+1]^{(2 g-2)}}$. By the trace we mean that $\mathcal{Y} \mathcal{M}(\alpha * \beta)=\mathcal{Y} \mathcal{M}(\beta * \alpha)$ for all $\alpha, \beta$. This trace is called the Yang Mills measure. If $t= \pm 1$ or $|t| \neq 1$, there is a diffeomorphism invariant, locally defined trace on $K_{t}(F)$ so that when $F$ is closed and $g>1$, $\mathcal{Y} \mathcal{M}(\emptyset)=\sum_{i=0}^{\infty} \frac{1}{[i+1]^{(2 g-2)}}$, and when $g=1$ or when $F$ has boundary then $\mathcal{Y} \mathcal{M}(\emptyset)=1$, [3] . If the surface $F$ has boundary, the locality condition can be characterized in the following way. If $\kappa$ is a properly embedded arc in $F$, let $F^{\prime}$ be the result of cutting $F$ along $\kappa$. Let $N_{t}(F)$ be the linear subspace of $K_{t}(F)$ spanned by skeins that are represented by admissibly colored trivalent graphs that intersect $\kappa$ in a single point of transverse intersection, so that the edge intersecting $\kappa$ carries a nonzero label. Let $\iota: K_{t}\left(F^{\prime}\right) \rightarrow K_{t}(F)$ be the natural inclusion map. Then

$$
K_{t}(F)=\operatorname{im}(\iota) \oplus N_{t}(F) .
$$

Locality is the statement that $\mathcal{Y} \mathcal{M}$ is zero on $N_{t}(F)$ and its restriction to $\operatorname{im}(\iota)$ is equal to $\mathcal{Y} \mathcal{M}$ on $K_{t}\left(F^{\prime}\right)$.

Let $A$ be an annulus which is the result of removing a regular neighborhood of a point from the disk $D$, and let $s_{u}$ be the skein in $A$ obtained by coloring a core of the annulus with the $u$-th Jones-Wenzl idempotent. Given a skein $\alpha$ in $D$, perturb the links representing it so that they miss the removed disk to get a skein in $A$, denoted $\alpha^{\prime}$. For the remainder of this and in the next section, use subscripts to indicate the domain of $\mathcal{Y} \mathcal{M}$. It is proved in 3 , that

$$
\mathcal{Y} \mathcal{M}_{D}(\alpha)=\sum_{u=0}^{\infty} \Delta_{u} \mathcal{Y} \mathcal{M}_{A}\left(\alpha^{\prime} * s_{u}\right) \text {. }
$$

Although the sum is infinite only a finite number of terms are nonzero.

If $F$ is a closed surface of genus greater than 1 , let $F^{\prime}$ be the result of removing a regular neighborhood of a point from $F$. Let $\partial_{u}$ be a small zero framed knot parallel to the boundary of $F^{\prime}$, colored with the $u$-th Jones-Wenzl idempotent. For a skein $\alpha$ in $F$, let $\alpha^{\prime}$ be a skein in $F^{\prime}$ obtained by perturbing all the links in a representative of $\alpha$ to lie in $F^{\prime}$. Then

$$
\mathcal{Y} \mathcal{M}_{F}(\alpha)=\sum_{u=0}^{\infty} \Delta_{u} \mathcal{Y} \mathcal{M}_{F^{\prime}}\left(\alpha^{\prime} * \partial_{u}\right)
$$

The series is absolutely convergent $[3]$ if $|t| \neq 1$ and when $t= \pm 1$. 
When $F$ has genus $g=1$ we define the quantities as above except that the Yang-Mills measure is given by the formula,

$$
\mathcal{Y} \mathcal{M}_{F}(\alpha)=\lim _{n \rightarrow \infty} \frac{1}{n} \sum_{u=0}^{n} \Delta_{u} \mathcal{Y} \mathcal{M}_{F^{\prime}}\left(\alpha^{\prime} * \partial_{u}\right) \text {. }
$$

Recall that the Kauffman bracket skein module of a disjoint union of two 3manifolds is the tensor product of the modules of the pieces. In particular if a surface $F$ is a disjoint union of surfaces $F_{1}$ and $F_{2}$ then

$$
K_{t}(F)=K_{t}\left(F_{1}\right) \otimes K_{t}\left(F_{2}\right) .
$$

The Yang Mills measure is bilinear with respect to the disjoint union. That is, if $\alpha \in K_{t}(F)$, where $F=F_{1} \sqcup F_{2}$ and $\alpha=\alpha_{1} * \alpha_{2}$ whith $\alpha_{1} \in K_{t}\left(F_{1}\right)$ and $\alpha_{2} \in K_{t}\left(F_{2}\right)$ then $\mathcal{Y} \mathcal{M}_{t}(\alpha)=\mathcal{Y} \mathcal{M}_{t}\left(\alpha_{1}\right) \mathcal{Y} \mathcal{M}_{t}\left(\alpha_{2}\right)$.

\section{A formula for evaluating the Yang-Mills measure of a colored framed link}

We begin with notation for describing the geography of link diagrams. The projection of a link onto the surface decomposes it into faces, edges and vertices. Given a face $f$, at each of its vertices there are two edges lying on the boundary of $f$; one of them is an over, and the other an under-crossing. Associate $-\frac{1}{2}$ to the vertex when in the counter-clockwise order the under comes before the over-crossing edge. Otherwise assign $\frac{1}{2}$ to the vertex (see Figure 2). The gleam $g_{f}$ of the face is the sum of these assignments over all the vertices lying on the boundary of $f$. The Euler characteristic of the face $f$ is denoted $x_{f}$.

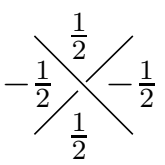

Figure 2: Contributions to the gleam at a vertex

Each edge $e$ lies between two faces $f_{e, 1}$ and $f_{e, 2}$. However, not every edge must belong to a vertex. Let $v(e)=1$ if $e$ is adjacent to some vertex, and $v(e)=0$ otherwise. The edge $e$ carries a color $k_{e}$ inherited from the coloring of the link.

At each vertex $v$ there are four edges corresponding to at most two components of the link, the over-crossing one colored with $k_{v, o}$, and the under-crossing one colored with $k_{v, u}$. Any vertex $v$ is adjacent to four corners of faces, labeled 


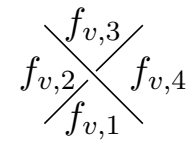

Figure 3: Labeling faces around a vertex

clockwise $f_{v, 1}, f_{v, 2}, f_{v, 3}$ and $f_{v, 4}$, so that the under-crossing edge lies between $f_{v, 1}$ and $f_{v, 2}$ as pictured in Figure 3 .

An admissible coloring of the diagram is an assignment of a color $u_{f} \in \mathbb{N}$ to each face $f$ so that along each edge the colors $u_{f_{e, 1}}, u_{f_{e, 2}}$, and $k_{e}$ form an admissible triple. Figure 4 shows a piece of a colored link diagram. The edges carry colors $1,2,3$ and 4 . The vertex $v$ has $k_{v, o}=1$ and $k_{v, u}=2$. Note that admissibility forces $u_{f_{1}}$ to be equal $u_{f_{4}}-2, u_{f_{4}}$ or $u_{f_{4}}+2$. The gleam of $f_{4}$ is 1 .

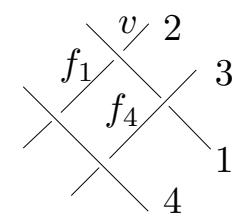

Figure 4: Fragment of a colored link diagram

Theorem 1 Let $F$ be a closed surface of genus $g>1$, and $\mathcal{L}$ a framed colored link in $F \times I$, whose diagram has faces $f$, edges $e$ and vertices $v$. Suppose that $|t| \neq 1$ or $t= \pm 1$. The value of $\mathcal{Y M}: K_{t}(M) \rightarrow \mathbb{C}$ on the skein corresponding to $\mathcal{L}$ is given by

$$
\sum_{u_{f}}(-1)^{\sum_{f} g_{f} u_{f}} t^{g_{f} u_{f}\left(u_{f}+2\right)} \frac{\prod_{f} \Delta_{u_{f}}^{x_{f}} \prod_{v} \operatorname{Tet}\left(\begin{array}{lll}
u_{f_{v, 1}} & u_{f_{v, 2}} & k_{v, u} \\
u_{f_{v, 3}} & u_{f_{v, 4}} & k_{v, o}
\end{array}\right)}{\prod_{e} \theta\left(u_{f_{e, 1}}, u_{f_{e, 2}}, k_{e}\right)^{v(e)}}
$$

where the sum is over all admissible colorings $u_{f}$ of the faces.

Proof The computation of the Yang-Mills measure of a skein on a closed surface starts by removing a disk from the surface and then taking the sum of values of the Yang-Mills measure of an associated family of skeins on the punctured surface. The first step of the proof is to show that an arbitrary number of punctures can be used. In this way we obtain a multiply infinite sum whose value is the Yang-Mills measure. The second step is reducing this sum to the expression (111). 
We prove the first step in a sequence of lemmas.

Lemma 1 Let $\left\{p_{i}\right\}_{i=1}^{n}$ be a collection of points in the interior of a disk $D$. Denote by $D^{\prime}$ the planar surface obtained by removing disjoint regular neighborhoods of the points $p_{i}$ from $D$, and let $L \subset D \times[0,1]$ be a framed link whose diagram lies in $D^{\prime}$. For each $i=1, \ldots, n$, let $\partial_{i, u}$ be a blackboard framed knot parallel to the boundary of the neighborhood of $p_{i}$, colored with the $u$-th Jones-Wenzl idempotent. Then

$$
\mathcal{Y} \mathcal{M}_{D}(L)=\sum_{u_{1}=0}^{\infty} \cdots \sum_{u_{n}=0}^{\infty}\left(\prod_{i=1}^{n} \Delta_{u_{i}}\right) \mathcal{Y} \mathcal{M}_{D^{\prime}}\left(L * \prod_{i=1}^{n} \partial_{i, u_{i}}\right)
$$

Proof By induction on $n$. Even though the sum is infinite, only finitely many terms are nonzero. For $n=1$ this is identity (7). Suppose the lemma is true for $n-1$ points, and choose a proper arc $\kappa \subset D^{\prime}$ that separates point $p_{n}$ from $\left\{p_{1}, \ldots, p_{n-1}\right\}$. Let $D_{1}$ and $D_{2}$ be the components of $D \backslash \kappa$ with $\left\{p_{1}, \ldots, p_{n-1}\right\} \subset D_{1}$, and let $D_{1}^{\prime}, D_{2}^{\prime}$ be the corresponding components of $D^{\prime} \backslash \kappa$. Recall that by (6) , the skein $[L]$ corresponding to the link $L$ can be written as $\lambda_{0}+\lambda_{n}$, where $\lambda_{0} \in \operatorname{im}\left(\iota: K_{t}\left(D_{1}\right) \otimes K_{t}\left(D_{2}\right) \rightarrow K_{t}(D)\right)$, and $\lambda_{n} \in N_{t}(D)$. We can express $\lambda_{0}$ as

$$
\lambda_{0}=\iota\left(\sum_{j} \lambda_{1, j} \otimes \lambda_{2, j}\right) .
$$

Thus by locality and by linearity,

$$
\begin{aligned}
& \mathcal{Y} \mathcal{M}_{D}(L)= \\
& \sum_{j} \sum_{u_{1}=0}^{\infty} \cdots \sum_{u_{n}=0}^{\infty}\left(\prod_{i=1}^{n} \Delta_{u_{i}}\right) \mathcal{Y} \mathcal{M}_{D_{1}}\left(\lambda_{1, j} * \prod_{i=1}^{n-1} \partial_{i, u_{i}}\right) \mathcal{Y} \mathcal{M}_{D_{2}}\left(\lambda_{2, j} * \partial_{n, u_{n}}\right) .
\end{aligned}
$$

By the distributive law this is equal to

$$
\begin{aligned}
\sum_{j}\left(\sum_{u_{1}=0}^{\infty} \cdots \sum_{u_{n-1}=0}^{\infty} \prod_{i=1}^{n-1} \Delta_{u_{i}} \mathcal{Y} \mathcal{M}_{D_{1}^{\prime}}\left(\lambda_{1, j} * \prod_{i=1}^{n-1} \partial_{i, u_{i}}\right)\right) \\
\left(\sum_{u_{n}=0}^{\infty} \Delta_{u_{n}} \mathcal{Y} \mathcal{M}_{D_{2}^{\prime}}\left(\lambda_{2, j} * \partial_{n, u_{n}}\right)\right)
\end{aligned}
$$

By induction this is further equal to

$$
\sum_{j} \mathcal{Y} \mathcal{M}_{D_{1}}\left(\lambda_{1, j}\right) \mathcal{Y} \mathcal{M}_{D_{2}}\left(\lambda_{2, j}\right),
$$

which, by locality, is $\mathcal{Y} \mathcal{M}_{D}(L)$. 
Next, we generalize Lemma 1 to an arbitrary surface with boundary.

Lemma 2 Let $\left\{p_{i}\right\}_{i=1}^{n}$ be a collection of points in the interior of a compact oriented surface $F$ with boundary. Denote by $F^{\prime}$ the result of removing disjoint regular neighborhoods of the points $p_{i}$ from $F$, and let $L \subset F \times[0,1]$ be a framed link whose diagram lies in $F^{\prime}$. The collection of knots $\partial_{i, u_{i}}$ is defined as in Lemma 1, Then

$$
\mathcal{Y} \mathcal{M}_{F}(L)=\sum_{u_{1}=0}^{\infty} \cdots \sum_{u_{n}=0}^{\infty}\left(\prod_{i=1}^{n} \Delta_{u_{i}}\right) \mathcal{Y} \mathcal{M}_{F^{\prime}}\left(L * \prod_{i=1}^{n} \partial_{i, u_{i}}\right)
$$

Proof Choose a family of proper arcs (called cross-cuts) in $F$ which lie in $F^{\prime}$ and cut $F$ into a disk $D$. Using identity (6) to decompose the skein $[L]$, with respect to the crosscuts we get $[L]=\lambda_{0}+\lambda_{n}$ where

$$
\mathcal{Y} \mathcal{M}_{F^{\prime}}(L)=\mathcal{Y} \mathcal{M}_{D}\left(\lambda_{0}\right) \text {. }
$$

Now apply Lemma 1

Finally, we prove the analogous lemma for closed surfaces.

Lemma 3 Suppose that $F$ is a closed surface of genus $g>1$. Let $\left\{p_{i}\right\}_{i=1}^{n}$, $F^{\prime}, L$, and $\partial_{i, u_{i}}$ be as in Lemma 2, Then

$$
\mathcal{Y} \mathcal{M}_{F}(L)=\sum_{u_{1}=0}^{\infty} \cdots \sum_{u_{n}=0}^{\infty}\left(\prod_{i=1}^{n} \Delta_{u_{i}}\right) \mathcal{Y} \mathcal{M}_{F^{\prime}}\left(L * \prod_{i=1}^{n} \partial_{i, u_{i}}\right)
$$

Proof Let $p_{0}$ be a point in $F^{\prime}$ disjoint from the diagram of $L$, and $N\left(p_{0}\right)$ its regular neighborhood. By the definition of the Yang-Mills measure (8),

$$
\mathcal{Y} \mathcal{M}_{F}(L)=\sum_{u_{0}=0}^{\infty} \Delta_{u_{0}} \mathcal{Y} \mathcal{M}_{F \backslash N\left(p_{0}\right)}\left(L * \partial_{0, u_{0}}\right) .
$$

Now apply Lemma 2 .

Thus the value of the Yang-Mills measure of a link can be computed using an arbitrary number of punctures. In order to prove Theorem 1 choose one point $p_{i}$ in each face $f_{i}$ of the diagram of the link $L$. The next step is to apply locality in the multiply punctured surface $F^{\prime}$ to compute the measure.

Choose a system of cross-cuts joining the punctures of adjacent faces, along with $2 h+b-1$ cross-cuts in the interior of each face of genus $h$ and $b$ boundary 
components, to cut it down to a disk. Fuse in neighborhoods of cross-cuts (as in Figure 5) to write the skein $[L]$ as a linear combination of colored trivalent graphs, each intersecting every cross-cut at most once transversally. In Figure 5 the crosscuts are depicted by dotted horizontal lines joining the little circles (i.e. boundaries of the punctures). The middle vertical line is a strand of the link, colored with $k_{e}$, the left vertical line is a strand of the knot $\partial_{i}$ colored with $u_{i}$, and the right vertical line is a strand of the knot $\partial_{j}$ colored with $u_{j}$.

$$
\begin{aligned}
& \mathcal{Y} \mathcal{M}(\circ . . . . \circ)=\sum_{c} \frac{\Delta_{c}}{\theta\left(c, u_{i}, k_{e}\right)} \mathcal{Y M}(\circ|\ldots| \circ)
\end{aligned}
$$

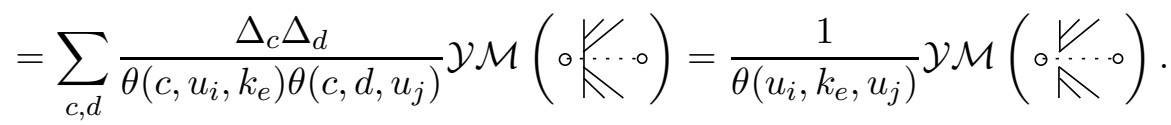

Figure 5: Fusing along cross-cuts.

The measure of $[L]$ is equal to the sum of the measures of those terms where every edge intersecting a cross-cut has color zero, with additional coefficients coming from fusion. These terms are unions of tetrahedra and theta curves. The tetrahedra correspond to the crossings of $L$, i.e., to the vertices of the diagram of $L$ (see figure 6). The theta curves come from strands of the link which are not involved in any crossings, i.e., from edges of the link diagram which are not adjacent to any vertices.

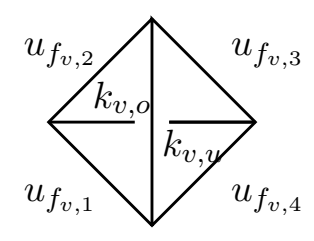

Figure 6: Tetrahedron around a vertex

Fusing along each of the $2 h+b-1$ cross-cuts for each face $f$ introduces the coefficient $\frac{1}{\theta\left(u_{f}, u_{f}, 0\right)}=\frac{1}{\Delta_{u_{f}}}$. Recalling the coefficient $\Delta_{u_{f}}$ coming from puncturing the face $f$ and totaling this with the contribution from the cross-cuts yields that the total factor coming from the face $f$ is $\left(\Delta_{u_{f}}\right)^{x_{f}}$.

Fusing along a cross-cut joining two faces $f_{1}, f_{2}$ that intersect along an edge $e$ introduces the coefficient $\frac{1}{\theta\left(u_{f_{e, 1},}, u_{\left.f_{e, 2}, k_{e}\right)}\right.}$. If $e$ is not adjacent to any vertex this coefficient cancels with the measure of the resulting theta curve. 
To compute the measure of a tetrahedron at the vertex $v$ slide the top edge in figure [6 to the side and use the tulip identity [12, Fig. 14.14]). This evaluates to

$$
(-1)^{n_{1}} t^{n_{2}} \operatorname{Tet}\left(\begin{array}{lll}
u_{f_{v, 1}} & u_{f_{v, 2}} & k_{v, u} \\
u_{f_{v, 3}} & u_{f_{v, 4}} & k_{v, o}
\end{array}\right)
$$

where $n_{1}=\frac{u_{f_{v, 1}}+u_{f_{v, 3}}-u_{f_{v, 2}}-u_{f_{v, 4}}}{2}$, and

$$
n_{2}=\frac{-u_{f_{v, 1}}\left(u_{f_{v, 1}}+2\right)-u_{f_{v, 3}}\left(u_{f_{v, 3}}+2\right)+u_{f_{v, 2}}\left(u_{f_{v, 2}}+2\right)+u_{f_{v, 4}}\left(u_{f_{v, 4}}+2\right)}{2} .
$$

Taking the product of all these, collecting the exponents of $t$ according to the faces, and collecting the powers of -1 according to the vertices, yields the desired formula.

Scholium 1 The analogous formula holds when $t=e^{\frac{\pi i}{2 r}}$ for odd $r \geq 3$, with the altered requirement that the colors $u_{f}$ be $r$-admissible.

Let $F$ be a compact oriented surface with boundary and $L$ a blackboard framed colored link in $F \times[0,1]$. An admissible coloring of a diagram of $L$ is an assignment of colors to the faces of the diagram so that each face containing a boundary component of $F$ carries the label 0 and along each edge the triple of colors coming from the edge and the adjacent faces is admissible.

Theorem 2 If $F$ is a compact oriented surface with boundary then the YangMills measure of a skein corresponding to a blackboard framed colored link can be computed using formula (11) where the sum is over all admissible colorings of a diagram corresponding to the link.

\section{The Shadow World}

Turaev [15] constructed a formalism for invariants of framed links in a circle bundle over a surface $F$. A shadow $S$ is a four-valent graph in a closed, oriented surface, with an assignment of an integer to each face of the graph, called the gleam of the face. The sum of all the gleams over all the faces is the total gleam of the shadow. Let $p: E \rightarrow F$ be a circle bundle. If $L \subset E$ is a link in general position with respect to $p$, then its image in $F$ is a four-valent graph. There is a recipe for deriving gleams from how the link lies in $E$. The total gleam is the negative of the Euler number of the circle bundle that the framed link lives in.

One can assign a shadow to a framed link in $F \times I$. Make sure it has the blackboard framing and then project it down to the surface. The graph is the 
image of the link under projection. The gleam of each face is assigned as in the computation of the Yang-Mills measure (see Figure 21). This coincides with the rule given in the Remark on page 41 of [15].

If the link is colored, the edges of its shadow inherit the colors. An admissible coloring of a shadow $S$ is an assignment of colors to the faces so that for each edge of the shadow the triple of colors from the adjacent faces and the edge is admissible. The formula for Turaev's shadow world evaluation of $S$ is a sum analogous to (11). However, there is no notion of over-crossing and under-crossing at a vertex. Hence for the gleams we use the assigned values $g_{f}$. Suppose that the colors of faces at a vertex are $u_{f_{v, 1}}, u_{f_{v, 2}}, u_{f_{v, 3}}$ and $u_{f_{v, 4}}$ going clockwise, with the edge between faces corresponding to $u_{f_{v, 1}}$ and $u_{f_{v, 2}}$, as well as the edge between faces corresponding to $u_{f_{v, 3}}$ and $u_{f_{v, 4}}$ colored $l_{v}$, and the other two edges colored $k_{v}$. Then the contribution for the vertex is

$$
\operatorname{Tet}\left(\begin{array}{lll}
u_{f_{v, 1}} & u_{f_{v, 2}} & k_{v} \\
u_{f_{v, 3}} & u_{f_{v, 4}} & l_{v}
\end{array}\right) \text {. }
$$

This is well defined because of the symmetry of the tetrahedral coefficients.

Let $\mathcal{T}(S)$ be the series,

$$
\begin{aligned}
\mathcal{T}(S)=\sum_{u_{f}}(-1)^{\sum_{f} g_{f} u_{f}} & \prod_{f} \Delta_{u_{f}}^{x_{f}} t_{f} u_{f}\left(u_{f}+2\right) . \\
& \prod_{e} \frac{1}{\theta\left(u_{f_{e, 1}}, u_{f_{e, 2}}, k_{e}\right)^{v(e)}} \prod_{v} \operatorname{Tet}\left(\begin{array}{lll}
u_{f_{v, 1}} & u_{f_{v, 2}} & k_{v} \\
u_{f_{v, 3}} & u_{f_{v, 4}} & l_{v}
\end{array}\right) .
\end{aligned}
$$

When $t$ is a $4 r$-th root of unity for odd $r$, the sum is finite, and $\mathcal{T}(S)$ is Turaev's evaluation corresponding to $U_{q}\left(s l_{2}\right)$.

Theorem 3 Suppose that $S$ is a shadow in a closed orientable surface $F$. If the total gleam of $S$ is positive then the series $\mathcal{T}(S)$ converges when $|t|<1$. If the total gleam is negative then the series converges when $|t|>1$. The series always converges when $t= \pm 1$ and the genus of $F$ is greater than 1 .

Proof Let $S$ be a shadow with positive gleam. The case of negative gleam is similar. We want to show that for $|t|<1$ the series (22) is absolutely convergent.

The fundamental estimate for tetrahedral coefficients [3] says that

$$
\left|\operatorname{Tet}\left(\begin{array}{lll}
a & b & e \\
c & d & f
\end{array}\right)\right| \leq \sqrt{\frac{\theta(b, c, e) \theta(a, d, e) \theta(a, b, f) \theta(c, d, f)}{(-1)^{e+f}[e+1][f+1]}} .
$$


Reorganizing the product according to vertices, the absolute values of the terms for $\mathcal{T}(S)$ become

$$
\begin{aligned}
& \prod_{f}\left|\Delta_{u_{f}}^{x_{f}} t_{f_{f} u_{f}\left(u_{f}+2\right)}\right| \cdot \\
& \prod_{v} \frac{\left|\operatorname{Tet}\left(\begin{array}{ccc}
u_{f_{v, 1}} & u_{f_{v, 2}} & k_{v} \\
u_{f_{v, 3}} & u_{f_{v, 4}} & l_{v}
\end{array}\right)\right|}{\sqrt{\left|\theta\left(u_{f_{v, 1}}, u_{f_{v, 2}}, k_{v}\right) \theta\left(u_{f_{v, 3}}, u_{f_{v, 4}}, k_{v}\right) \theta\left(u_{f_{v, 2}}, u_{f_{v, 3}}, l_{v}\right) \theta\left(u_{f_{v, 4}}, u_{f_{v, 1}}, l_{v}\right)\right|}}
\end{aligned}
$$

Applying the estimate (23), this is less than or equal to

$$
\prod_{f}\left|\Delta_{u_{f}}^{x_{f}} t^{g_{f} u_{f}\left(u_{f}+2\right)}\right| \prod_{v} \frac{1}{\sqrt{\left[k_{v}+1\right]\left[l_{v}+1\right]}} .
$$

Since the product over the vertices does not depend on the coloring of the faces, we factor it out to the front as a constant $C$. Now we have

$$
C \prod_{f}\left|\Delta_{u_{f}}^{x_{f}} t^{g_{f} u_{f}\left(u_{f}+2\right)}\right| \text {. }
$$

Let $B$ (the breadth) of the shadow be the maximum possible difference of the colors on any two faces under an admissible coloring. Given a particular coloring, let $U$ be the maximal color assigned to a face. Let $p$ be the sum of the gleams of the faces with positive gleam and $n$ be the sum of the gleams of the faces with negative gleam, so that $p+n$ is the total gleam of the shadow. By replacing all $u_{f}$ on the negative gleam faces by $U$ and all $u_{f}$ on the faces of positive gleam by $U-B$ we get,

$$
C \prod_{f}\left|\Delta_{u_{f}}^{x_{f}} t^{g_{f} u_{f}\left(u_{f}+2\right)}\right| \leq C \prod_{f}\left|\Delta_{u_{f}}^{x_{f}}\right| t^{n U(U+2)+p(U-B)(U-B+2)}
$$

We decompose the faces into two subsets, $F^{+}$consisting of faces with positive Euler characteristic, and $F^{-}$consisting of faces with negative Euler characteristic. The product (27) is less than or equal to:

$$
C\left|\frac{\prod_{f \in F^{+}} \Delta_{U}^{x_{f}}}{\prod_{f \in F^{-}} \Delta_{U-B}^{-x_{f}}}\right||t|^{(n+p) U(U+2)-2 p B U+2 p(U-B)+p B^{2}} .
$$

Rewrite (28) as,

$$
C \frac{1}{\left|\Delta_{U-B}\right|^{-(2-2 g)}} \prod_{f \in F^{+}}\left|\frac{\Delta_{U}}{\Delta_{U-B}}\right|^{x_{f}}|t|^{(n+p) U(U+2)-2 p B U+2 p(U-B)+p B^{2}}
$$


Since $|t|^{-2 n} \leq\left|\Delta_{n}\right| \leq(n+1)|t|^{-2 n}$

$$
\frac{1}{\left|\Delta_{U-B}^{-(2-2 g)}\right|} \leq|t|^{2 U(2-2 g)}
$$

and

$$
\prod_{f \in F^{+}}\left(\frac{\left|\Delta_{U}\right|}{\left|\Delta_{U-B}\right|}\right)^{x_{f}}=\left(\frac{\left|\Delta_{U}\right|}{\left|\Delta_{U-B}\right|}\right)^{p} \leq U^{p}|t|^{-2 B p} .
$$

Thus (29) is less than or equal to

$$
C U^{p}|t|^{2 U(2-2 g)-2 B p+(n+p) U(U+2)-2 p B U+2 p(U-B)+p B^{2}} .
$$

Since $n+p>0$ the exponent is a quadratic function in $U$ with positive lead coefficient. Therefore, as $U$ gets large, this is eventually smaller than the terms of a geometric series.

Now reorganize the terms in the sum for $\mathcal{T}(S)$ according to the maximal color $U$. There are at most $B^{\# \text { faces }}$ admissible colorings having the same $U$. Thus,

$$
\mathcal{T}(S) \leq \sum_{U} B^{\# f} C U^{p}|t|^{2 U(2-2 g)-2 B p+(n+p) U(U+2)-2 p B U+2 p(U-B)+p B^{2}}
$$

which is clearly convergent.

Remark 1 There exist shadows of total gleam 0 for which $\mathcal{T}(S)$ is never convergent when $|t| \neq 1$.

A simple example is obtained by a separating non null-homotopic closed curve on a surface of genus 2 with gleams on the faces equal to 4 and -4 .

\section{$5 \quad$ Limiting behavior at $t=-1$}

In 3 we showed that as $t \rightarrow-1$ from the interior or the exterior of the unit disk then the Yang-Mills measure converges to the Yang-Mills measure at $t=-1$. It is the goal of this section to show that, as $r \rightarrow \infty$, the Yang-Mills measure at the $4 r$-th root of unity, $\mathcal{Y M}_{r}: K_{r}(F) \rightarrow \mathbb{C}$, converges to twice the Yang-Mills measure at $t=-1, \mathcal{Y} \mathcal{M}_{-1}: K_{-1}(F) \rightarrow \mathbb{C}$. Although it can be shown using the Symmetry Principle for the shadow world formula, we can see this more simply using the formulas from [3]. Note that in this section, subscripts of $\mathcal{Y} \mathcal{M}$ are used to indicate the value of the complex parameter $t$. 
Remark 2 The Yang-Mills measure is not continuous in the variable $t$ at nontrivial $4 r$-th roots of unity.

For instance, let $F$ be an annulus and take $s$ to be the skein determined by 4 parallel copies of the central core. Then for $|t|<1, \mathcal{Y M}_{t}(s)=2$, while at the 12-th root of unity $\mathcal{Y M}_{3}(s)=1$.

Theorem 4 Let $F$ be a closed surface of genus greater than 1. Given a colored framed link $L$ in $F \times I$,

$$
\lim _{r \rightarrow \infty} \mathcal{Y M}_{r}(L)=2 \cdot \mathcal{Y} \mathcal{M}_{-1}(L)
$$

Proof The proof requires the use of the following Symmetry Principle for the Kauffman bracket.

Proposition 1 Let $L$ be a framed link, and $K$ a framed knot disjoint from $L$, with framing $f$. Denote by $K_{u}$ the knot $K$ colored with $u$, and by $\langle L\rangle$ the value of the Kauffman bracket of $L$ evaluated at the $4 r$-th root of unity. Then

$$
\left\langle L \cup K_{u}\right\rangle=i^{f(r+2 u-2)}(-1)^{1+\lambda}\left\langle L \cup K_{r-u-2}\right\rangle,
$$

where $\lambda=\sum \operatorname{lk}\left(L_{i}, K\right)$, and the sum is taken over all components $L_{i}$ of the link $L$ carrying odd colors.

In particular, if the framing on the component $K$ is 0 then

$$
\left\langle L \cup K_{u}\right\rangle=(-1)^{1+\lambda}\left\langle L \cup K_{r-u-2}\right\rangle .
$$

Proof It follows immediately from the Symmetry Principle proved in [10]. Notice that their color $k$ corresponds to our color $k-1$.

To compute the Yang-Mills measure of the skein $s$ in $K_{r}(F)$, first puncture the surface, to get a surface $F^{\prime}$ with boundary, and then take the sum over all the labels $u$ on the blackboard framed knot $\partial$ parallel to the boundary [3] as follows:

$$
\mathcal{Y} \mathcal{M}_{r}(s)=\sum_{u=0}^{r-2} \Delta_{u} \mathcal{Y} \mathcal{M}_{r}\left(\partial_{u} * s\right) .
$$

We are using the subscript $r$ in $\mathcal{Y} \mathcal{M}_{r}$ to emphasize the fact that the parameter $t$ is a $4 r$-th root of unity. Here $\mathcal{Y} \mathcal{M}_{r}$ on the right hand side is the Yang-Mills 
measure taken in the punctured surface $F^{\prime}$. Proposition 1 implies that (37) is equal to

$$
2 \sum_{u=0}^{\frac{r-3}{2}} \Delta_{u} \mathcal{Y} \mathcal{M}_{r}\left(\partial_{u} * s\right)
$$

Let $\gamma$ be a trivalent spine of the surface $F^{\prime}$. Recall that any skein in $K_{r}\left(F^{\prime}\right)$ can be written as a linear combination of the skeins corresponding to the $r$ admissible colorings of $\gamma$. Since the coefficients are continuous as $r \rightarrow \infty$, it is enough to show that

$$
\lim _{r \rightarrow \infty} \mathcal{Y} \mathcal{M}_{r}\left(s_{c}\right)=2 \mathcal{Y} \mathcal{M}_{-1}\left(s_{c}\right)
$$

where $s_{c}$ is a skein coming from the coloring $c$ of $\gamma$.

Suppose that $\gamma$ has edges $e$ and vertices $v$. Let $k_{e}$ denote the color associated to the edge $e$ in $s_{c}$, and $k_{v, 1}, k_{v, 2}, k_{v, 3}$ be the colors of the three edges ending in a vertex $v$. Using the formula for $\mathcal{Y M}_{r}\left(s_{c}\right)$ from [3] together with (38) we get:

$$
\mathcal{Y} \mathcal{M}_{r}\left(s_{c}\right)=2 \sum_{u=0}^{\frac{r-3}{2}} \Delta_{u} \prod_{e} \frac{1}{\theta\left(u, u, k_{e}\right)} \prod_{v} \operatorname{Tet}\left(\begin{array}{ccc}
u & u & u \\
k_{v, 1} & k_{v, 2} & k_{v, 3}
\end{array}\right) .
$$

Fix $\epsilon>0$. We will show that there exists $K>0$ so that for all $r$,

$$
\left|2 \sum_{u=K}^{\frac{r-3}{2}} \Delta_{u} \prod_{e} \frac{1}{\theta\left(u, u, k_{e}\right)} \prod_{v} \operatorname{Tet}\left(\begin{array}{ccc}
u & u & u \\
k_{v, 1} & k_{v, 2} & k_{v, 3}
\end{array}\right)\right|<\frac{\epsilon}{4},
$$

and that the analogous inequality, with no upper bound on the summation, holds for $t=-1$. Henceforth we will write the summation without any upper bounds, as the computation is similar at $t=-1$ and at $t=e^{\frac{\pi i}{2 r}}$. The first step is to rewrite the summands of (41) in terms of the vertices, so it becomes,

$$
2 \sum_{u \geq K}\left|\Delta_{u}\right| \prod_{v} \frac{\left|\operatorname{Tet}\left(\begin{array}{ccc}
u & u & u \\
k_{v, 1} & k_{v, 2} & k_{v, 3}
\end{array}\right)\right|}{\sqrt{\left|\theta\left(u, u, k_{v, 1}\right) \theta\left(u, u, k_{v, 2}\right) \theta\left(u, u, k_{v, 3}\right)\right|}} .
$$

From the fundamental estimate (23) we see that (42) is less than or equal to

$$
2 \sum_{u \geq K}\left|\Delta_{u}\right| \prod_{v} \frac{1}{\sqrt{\left|\Delta_{u}\right|}}=2 \sum_{u \geq K} \frac{1}{[u+1]^{2 g-2}},
$$

since the number of vertices of $\gamma$ is equal to $4 g-2$. As $[n] \geq \frac{\pi}{2} n$, the quantity (43) is less than or equal to

$$
\left(\frac{2}{\pi}\right)^{2 g-2} \sum_{u \geq K} \frac{1}{(n+1)^{2 g-2}}
$$


which is smaller than $\frac{\epsilon}{4}$ for big enough $K$.

Given $K$,

$$
\left|2 \sum_{u=0}^{K} \Delta_{u} \mathcal{Y} \mathcal{M}_{r}\left(\partial_{u} * s\right)-2 \sum_{u=0}^{K} \Delta_{u} \mathcal{Y} \mathcal{M}_{-1}\left(\partial_{u} * s\right)\right| \leq \frac{\epsilon}{2}
$$

if $r$ is big enough. Thus

$$
\begin{aligned}
\mid \mathcal{Y} \mathcal{M}_{r}\left(s_{c}\right)- & 2 \mathcal{Y} \mathcal{M}_{-1}\left(s_{c}\right) \mid \\
\leq & \left|2 \sum_{u=0}^{K} \Delta_{u} \mathcal{Y} \mathcal{M}_{r}\left(\partial_{u} * s\right)-2 \sum_{u=0}^{K} \Delta_{u} \mathcal{Y} \mathcal{M}_{-1}\left(\partial_{u} * s\right)\right| \\
+ & \left|2 \sum_{u=K}^{\frac{r-3}{2}} \Delta_{u} \prod_{e} \frac{1}{\theta\left(u, u, k_{e}\right)} \prod_{v} \operatorname{Tet}\left(\begin{array}{ccc}
u & u & u \\
k_{v, 1} & k_{v, 2} & k_{v, 3}
\end{array}\right)\right| \\
& +\left|2 \sum_{u=K}^{\infty} \Delta_{u} \mathcal{Y} \mathcal{M}_{-1}\left(\partial_{u} * s\right)\right| \leq \epsilon .
\end{aligned}
$$

\section{Integrating on the character variety}

We will use the formula from Theorem 11 to compute some examples. The irreducible $S U(2)$-characters of $\pi_{1}(F)$ will be denoted $\operatorname{char}^{i}\left(\pi_{1}(F)\right)$. Recall that the algebra $K_{-1}(F)$ is isomorphic to the ring of polynomial functions on $\operatorname{char}^{i}\left(\pi_{1}(F)\right)$, 1, 13]. The Yang-Mills measure is the linear functional induced by integrating against the symplectic measure 3 . Given a diagram on $F$ it corresponds to a function on $\operatorname{char}^{i}\left(\pi_{1}(F)\right)$. Under this correspondence a knot on a surface is taken to the function that assigns to a representation $\rho$ the negative of the trace of a loop determined by the knot in $\pi_{1}(F)$. In addition, any colored diagram represents an element of $H_{1}\left(F ; \mathbb{Z}_{2}\right)$ by replacing each edge

colored with $n$ by $n$ parallel strands, hooked together at trivalent vertices as if they were triads.

Proposition 2 If the diagram $\alpha$ does not represent 0 in $H_{1}\left(F ; \mathbb{Z}_{2}\right)$ then integrating $\alpha$ over $\operatorname{char}^{i}\left(\pi_{1}(F)\right)$ yields zero. In particular, if $\alpha$ is a non-separating curve, then the integral of $\alpha$ is 0 . 
Proof The diagram of $\alpha$ has no admissible colorings. If $\alpha$ represents a nonzero element of $H_{1}\left(F ; \mathbb{Z}_{2}\right)$ then there is a path $\gamma$ in $F$ which has non-zero intersection number with the cycle represented by $\alpha$. In an admissible coloring, the difference of the colors on two adjacent faces is congruent modulo 2 to the color of the edge between them. Hence the intersection number of $\alpha$ and $\gamma$ is the sum of the differences of the colors of the faces that $\gamma$ passes through. This contradicts the fact that $\gamma$ has a nonzero $\mathbb{Z}_{2}$-intersection number with $\alpha$.

Proposition 3 Let $\beta$ be a simple closed curve which separates the closed surface $F$ into surfaces of genus $G_{1}>0$ and $G_{2}>0$ respectively. Integrating $\beta$ yields

$$
\mathcal{Y} \mathcal{M}(\beta)=-\sum_{u=1}^{\infty}\left(\frac{1}{u^{2 G_{1}-1}(u+1)^{2 G_{2}-1}}+\frac{1}{(u+1)^{2 G_{1}-1} u^{2 G_{2}-1}}\right) .
$$

Proof The diagram of $\beta$ has two faces, $f_{1}$ and $f_{2}$, meeting along the only edge $e$ colored with 1 . There are no vertices, so $v(e)=0$. Let $u_{i}$ be the color assigned to $f_{i}$, for $i=1,2$. In order for the triple $\left(u_{1}, u_{2}, 1\right)$ to be admissible, we must have $u_{1}=u_{2}-1$ or $u_{1}=u_{2}+1$. Thus we have a sum over all nonnegative integers $u$ with two terms: one where $u_{1}=u$ and $u_{2}=u+1$, and one where $u_{1}=u+1$ and $u_{2}=u$. The Euler characteristics of the faces are $x_{1}=1-2 G_{1}$ and $x_{2}=1-2 G_{2}$. The gleams $g_{1}$ and $g_{2}$ of both faces are zero.

When $t=-1$, all the quantized integers in our formulas are replaced by ordinary integers. We have:

$$
\begin{aligned}
& \mathcal{Y} \mathcal{M}(\beta)= \\
& \sum_{u}\left(\Delta_{u}^{1-2 G_{1}} \Delta_{u+1}^{1-2 G_{2}} \frac{1}{(\theta(u, u+1,1))^{0}}+\Delta_{u+1}^{1-2 G_{1}} \Delta_{u}^{1-2 G_{2}} \frac{1}{(\theta(u+1, u, 1))^{0}}\right)
\end{aligned}
$$

Note that $\Delta_{u}=(-1)^{u}(u+1)$.

Substituting we get

$$
\begin{aligned}
& \mathcal{Y M}(\beta)= \\
& \begin{array}{l}
\sum_{u}\left((-1)^{p_{1}} \frac{1}{(u+1)^{2 G_{1}-1}(u+2)^{2 G_{2}-1}}+(-1)^{p_{2}} \frac{1}{(u+2)^{2 G_{1}-1}(u+1)^{2 G_{2}-1}}\right) \\
\quad=-\sum_{u=1}^{\infty}\left(\frac{1}{u^{2 G_{1}-1}(u+1)^{2 G_{2}-1}}+\frac{1}{(u+1)^{2 G_{1}-1} u^{2 G_{2}-1}}\right),
\end{array}
\end{aligned}
$$

since $p_{1}=u\left(1-2 G_{1}\right)+(u+1)\left(1-2 G_{2}\right)$ and $p_{2}=(u+1)\left(1-2 G_{1}\right)+u\left(1-2 G_{2}\right)$ are both odd. 
We can express $\mathcal{Y} \mathcal{M}(\beta)$ in terms of Bernoulli numbers.

Corollary 1 Suppose that $G_{1}=G_{2}=G$. Then

$$
\mathcal{Y} \mathcal{M}(\beta)=-2 \sum_{j=1}^{2 G-1}\left(\begin{array}{l}
4 G-j-3 \\
2 G-j-1
\end{array}\right)+4 \sum_{j=1}^{G-1}\left(\begin{array}{l}
4 G-2 j-3 \\
2 G-2 j-1
\end{array}\right) \frac{1}{2}\left|B_{2 j}\right| \frac{(2 \pi)^{2 j}}{(2 j) !}
$$

Otherwise let $G=\min \left(G_{1}, G_{2}\right)$, and $H=\max \left(G_{1}, G_{2}\right)$. Then

$$
\begin{gathered}
\mathcal{Y} \mathcal{M}(\beta)=-\sum_{j=1}^{2 G-1}\left(\begin{array}{c}
2 G+2 H-j-3 \\
2 G-j-1
\end{array}\right)-\sum_{j=1}^{2 H-1}\left(\begin{array}{c}
2 G+2 H-j-3 \\
2 H-j-1
\end{array}\right)+ \\
2 \sum_{i=1}^{G-1}\left(\left(\begin{array}{c}
2 G+2 H-2 i-3 \\
2 G-2 i-1
\end{array}\right)+\left(\begin{array}{c}
2 G+2 H-2 i-3 \\
2 H-2 i-1
\end{array}\right)\right) \frac{1}{2}\left|B_{2 i}\right| \frac{(2 \pi)^{2 i}}{(2 i) !} \\
+2 \sum_{i=G}^{H-1}\left(\begin{array}{c}
2 G+2 H-2 i-3 \\
2 H-2 i-1
\end{array}\right) \frac{1}{2}\left|B_{2 i}\right| \frac{(2 \pi)^{2 i}}{(2 i) !} .
\end{gathered}
$$

Proof Collect the terms and note that if $r$ is even, then $\sum \frac{1}{n^{r}}=\frac{1}{2}\left|B_{r}\right| \frac{(2 \pi)^{r}}{r !}$ where $B_{r}$ is a Bernoulli number.

We finish by giving numerical values in Figure 7 for the Yang-Mills measure of separating curves on surfaces with genus up to 10 . Here $G=G_{1}+G_{2}$ is the genus of the surface, the curve splits it into surfaces of genus $G_{1}$ and $G_{2}$ respectively. 


\begin{tabular}{|l|l|l|l|}
\hline $\mathrm{G}$ & $G_{1}$ & $G_{2}$ & Value of the integral \\
\hline 2 & 1 & 1 & -2 \\
\hline 3 & 1 & 2 & $-4+\frac{1}{3} \pi^{2}$ \\
\hline 4 & 1 & 3 & $-6+\frac{1}{45} \pi^{4}+\frac{1}{3} \pi^{2}$ \\
\hline 5 & 1 & 4 & $-8+\frac{2}{945} \pi^{6}+\frac{1}{45} \pi^{4}+\frac{1}{3} \pi^{2}$ \\
\hline 6 & 1 & 5 & $-10+\frac{1}{4725} \pi^{8}+\frac{2}{945} \pi^{6}+\frac{1}{45} \pi^{4}+\frac{1}{3} \pi^{2}$ \\
\hline 4 & 2 & 2 & $-20+2 \pi^{2}$ \\
\hline 5 & 2 & 3 & $-56+\frac{1}{15} \pi^{4}+5 \pi^{2}$ \\
\hline 6 & 2 & 4 & $-120+\frac{2}{315} \pi^{6}+\frac{2}{9} \pi^{4}+\frac{28}{3} \pi^{2}$ \\
\hline 7 & 2 & 5 & $-220+\frac{1}{1575} \pi^{8}+\frac{4}{189} \pi^{6}+\frac{7}{15} \pi^{4}+15 \pi^{2}$ \\
\hline 6 & 3 & 3 & $-252+\frac{2}{9} \pi^{4}+\frac{70}{3} \pi^{2}$ \\
\hline 7 & 3 & 4 & $-792+\frac{2}{189} \pi^{6}+\frac{14}{15} \pi^{4}+70 \pi^{2}$ \\
\hline 8 & 3 & 5 & $-2002+\frac{1}{945} \pi^{8}+\frac{2}{27} \pi^{6}+3 \pi^{4}+165 \pi^{2}$ \\
\hline 8 & 4 & 4 & $-3432+\frac{4}{135} \pi^{6}+\frac{56}{15} \pi^{4}+308 \pi^{2}$ \\
\hline 9 & 4 & 5 & $-11440+\frac{1}{675} \pi^{8}+\frac{62}{315} \pi^{6}+\frac{209}{15} \pi^{4}+1001 \pi^{2}$ \\
\hline 10 & 5 & 5 & $-48620+\frac{2}{525} \pi^{8}+\frac{44}{63} \pi^{6}+\frac{286}{5} \pi^{4}+4290 \pi^{2}$ \\
\hline 5 & 1 & & \\
\hline & & &
\end{tabular}

Figure 7: Integrals of separating curves on a closed surface of genus $G$ 


\section{References}

[1] D. Bullock, Rings of $S L_{2}(\mathbb{C})$-characters and the Kauffman bracket skein module, Comment. Math. Helv. 72 (1997), 521-542.

[2] D. Bullock, C. Frohman, J. Kania-Bartoszynska, Understanding the Kauffman Bracket Skein Module, J. Knot Theory Ramifications 8 (1999), no. 3, 265-277.

[3] D. Bullock, C. Frohman, J. Kania-Bartoszynska, The Yang-Mills measure in the Kauffman bracket skein module, Comment. Math. Helv. 78 (2003), 1-17.

[4] C. Frohman, Natural Volumes on Character Varieties and the Turaev-Viro Invariant at Level Infinity, in preparation.

[5] C. Frohman, J. Kania-Bartoszynska, The Quantum Content of the Normal Surfaces in a Three-Manifold, preprint.

[6] W. M. Goldman, The symplectic nature of fundamental groups of surfaces, Advances in Mathematics 54 (1984), no. 2, 200-225.

[7] V. F. R. Jones, A polynomial invariant for knots via von Neumann algebras, Bull. Amer. Math. Soc. 12, (1985) 103-111.

[8] L. H. Kauffman, State models and the Jones polynomial, Topology 26, no. 3 (1987) 395-401.

[9] L. H. Kauffman and S. Lins, Temperley-Lieb recoupling theory and invariants of 3 -manifolds, Ann. of Math. Studies 143, Princeton University Press (1994).

[10] R. Kirby, P. Melvin, The 3-manifold invariants of Witten and ReshetikhinTuraev for $\operatorname{sl}(2, \mathbb{C})$, Invent. Math. 105, (1991) 473-545.

[11] A. N. Kirillov, N. Yu. Reshetikhin, Representations of the algebra $U_{q}\left(s l_{2}\right)$, qorthogonal polynomials and invariants of links, Infinite Dimensional Lie Algebras and Groups (V. G. Kac, ed.), Advanced Ser. in Math. Phys., Vol 7, 1988, $285-339$.

[12] W. B. R. Lickorish, An Introduction to Knot Theory, Springer, GTM 175, 1997.

[13] J. H. Przytycki, A. S. Sikora, On skein algebras and $S L_{2}(\mathbb{C})$-character varieties, Topology 39, no 1, (2000), 115-148.

[14] J. Roberts, Skein theories as TQFTs, preprint.

[15] V. G. Turaev, Shadow links and face models of statistical mechanics, J. Diff. Geom. 36 (1992), 35-74.

[16] H. Wenzl, On sequences of projectors, C.R. Math. Rep. Acad. Sci. IX, (1987) $5-9$.

Department of Mathematics, University of Iowa, Iowa City, IA 52242, USA

and

Department of Mathematics, Boise State University, Boise, ID 83725, USA

Email: frohman@math.uiowa.edu and kania@math.boisestate.edu

URL: http://www.math.uiowa.edu/ ${ }^{\text {frohman }}$ and

http://math.boisestate.edu/ Kania

Algebraic 83 Geometric Topology, Volume 4 (2004) 